\title{
Recurrent Upper Quadrant Pain: A Fish Bone Secondary to Gastric Perforation and Liver Abscess
}

\author{
Hui Liang ${ }^{a}$ Ou-Qi Liu ${ }^{b}$ Xin-Bo Ai ${ }^{b}$ Da-Qi Zhu \\ Jin-Lan Liu ${ }^{b}$ An Wang ${ }^{b}$ Fei-Yue Gong ${ }^{b}$ Cong Hu ${ }^{a}$ \\ Departments of a General Surgery and ${ }^{b}$ Gastroenterology, The Third Affiliated \\ Hospital of Jinan University College of Medicine, Zhuhai People's Hospital, \\ Zhuhai, China
}

\section{Key Words}

Liver abscess · Fish bone · Coronary artery disease · Gastric ulcer

\begin{abstract}
A 60-year-old male patient was admitted to our hospital for recurrent upper quadrant pain for 1 month. He had a past history of coronary artery disease. After admission, he repeatedly suffered from high-grade fever, chills and upper quadrant pain. Computed tomography (CT) showed a round hypodense mass in the left lobe of the liver, approximately $2.7 \times 2.2 \mathrm{~cm}$ in size, and a fish bone was confirmed by surgery in the left lobe of liver. The patient was cured completely after surgical removal of the fish bone and liver abscess. CT scan 1 month after discharge showed that the liver abscess had disappeared completely.
\end{abstract}

\section{Introduction}

Gastrointestinal perforation by ingested fish bone resulting in hepatic abscess is relatively uncommon [1]. Most patients do not recall swallowing foreign bodies, and the clinical diagnosis may be rather difficult due to nonspecific clinical presentations [2]. We report a 60-year-old male patient who was admitted to our hospital for upper quadrant pain, diagnosed as coronary artery disease and peptic ulcer disease. However, the patient subsequently presented with recurrent high-grade fever and chills. Computed tomography (CT) showed a round hypodense mass in the left lobe of liver, approximately $2.7 \times 2.2 \mathrm{~cm}$ in size, and a fish bone was confirmed by surgery in the left lobe of the liver. The patient's clinical symptoms alleviated completely after surgical removal of the fish bone and the liver abscess. 


\section{Case Report}

A 60-year-old male patient presented with recurrent upper quadrant pain for 1 month. He had a medical history of coronary artery disease for 20 days. Coronary angiography revealed $65 \%$ stenosis of the left anterior descending branch of the coronary artery. The patient had suffered from gout for 1 year due to right ankle pain. He also had an 18-year upper gastrointestinal bleeding history. Endoscopy revealed duodenal ulcer at the anterior wall of the bulb. On physical examination at admission, he had a temperature of $36.5^{\circ} \mathrm{C}$, a heart rate of $60 \mathrm{bpm}$, a respiratory rate of 20 per minute, and a blood pressure of $160 / 100 \mathrm{~mm} \mathrm{Hg}$. Upper quadrant tenderness was found but without peritoneal signs. Laboratory examinations showed the following: white blood cells $16.6 \times 10^{9} / \mathrm{l}$, serum C-reactive protein $40 \mathrm{~g} / \mathrm{ml}$, erythrocyte sedimentation rate $40 \mathrm{~mm} / \mathrm{h}, \mathrm{CK}-\mathrm{MB} 13 \mathrm{U} / \mathrm{l}$, CTNI <0.01 $\mu \mathrm{g} / \mathrm{l}$, CK $582 \mathrm{U} / \mathrm{l}$, serum bilirubin $28.1 \mu \mathrm{mol} / \mathrm{l}$; aspartate aminotransferase and alanine aminotransferase were below normal level. An electrocardiogram revealed ST segment elevation in leads V1-V4. On the second day after admission, his temperature rose rapidly to a maximal level of $39.0^{\circ} \mathrm{C}$. Given his painful right ankle, his past history of gout and fluctuating temperature, he was prescribed nonsteroidal anti-inflammatory drugs as painkillers, omeprazole injection $40 \mathrm{mg}$ i.v. gtt bid for alleviating abdominal pain due to past duodenal ulcer, and nitroglycerin tablets for dilating the coronary artery. Consequently, his temperature decreased to a normal level of $36.8^{\circ} \mathrm{C}$. However, it rose sharply to $40.0^{\circ} \mathrm{C} 1$ week later. CT showed a round hypodense mass in the left lobe of liver, approximately $2.7 \times 2.2 \mathrm{~cm}$ in size, and a linear foreign body with hyperdensity lesion in the left lobe of the liver (fig. 1). Nasal endoscopy (Fujinon EG-530WN, Tokyo, Japan) showed an ulcer $0.9 \times 1.0 \mathrm{~cm}$ in size at the lesser curve of the gastric antrum (fig. 2). The patient was transferred to the Department of General Surgery with a fish bone in the left lobe of the liver (fig. 3 ) and a fistula between the stomach and the liver. The fish bone was removed, and the fistula lesion was cut successfully. Meanwhile, a percutaneous drainage tube was implanted at the abscess lesion of left lobe of the liver. As a result, the patient's symptoms or syndromes alleviated completely with surgery and antibiotics prescription.

\section{Discussion}

Gastrointestinal perforation is commonly caused by foreign bodies, such as fish bones, chicken bones, needles and toothpicks. The duodenum and pylorus may be more susceptible to perforation [3]. In our case, gastric antrum perforation was caused by a fish bone. This is not reported commonly.

Liver abscess is very familiar to clinicians. Its clinical manifestations are varied. Some patients present with high-grade fever, right upper quadrant pain and jaundice. However, other patients are not specific [4], so the diagnosis of liver abscess is rather difficult. Contrast-enhanced CT scan is of great importance in detecting the abscess. When it comes to the etiology of liver abscess secondary to fish bone, a possible pathogenesis is penetration through the duodenum and migration from the posterior surface of the liver into the liver [5]. Surgery is the mainstay treatment for liver abscess and foreign body ingestion [6]. Our patient experienced recurrent abdominal pain and intermittent fever; he could not remember whether he had a history of fish bone ingestion. CT showed a foreign body and a round hypodense mass located in the left lobe of the liver. The patient was cured completely after surgical removal of the fish bone and the liver abscess.

Coronary artery disease is very commonly found in elderly patients. Some patients may present with upper quadrant pain or bleeding [7]. Our patient underwent coronary angiography due to upper quadrant pain and ST segment elevation in leads $\mathrm{V} 1-\mathrm{V} 4$, and this revealed 65\% stenosis of the left anterior descending branch of the coronary artery. However, the patient still suffered upper quadrant pain, and 
high-grade temperature was observed subsequently. Upper quadrant pain accompanied by recurrent fever should be possibly considered as foreign body ingestion, and CT scan should be performed simultaneously.

In conclusion, we report a patient with liver abscess presenting with upper quadrant pain and high-grade fever; the liver abscess was secondary to fish bone ingestion. We should be aware of foreign body ingestion if patients present with abdominal pain and intermittent or persistent fever.
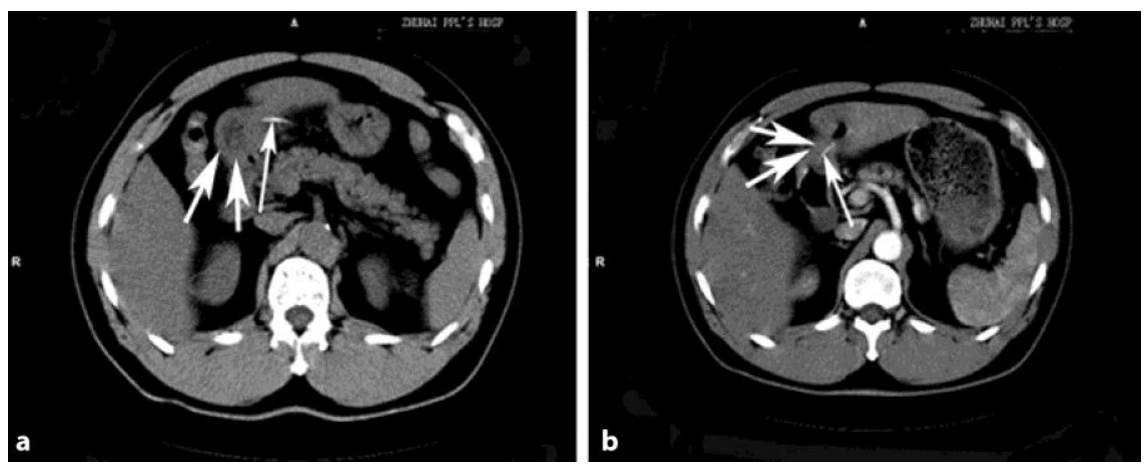

Fig. 1. a CT scan image showing a linear foreign body with hyperdense lesion in the left lobe of the liver (long arrow) and a round hypodense mass in the left lobe of the liver approximately $2.7 \times 2.2 \mathrm{~cm}$ in size (short arrows). b Contrast-enhanced CT scan indicating a linear foreign body with hyperdense lesion in the left lobe of the liver (long arrow) and a round hypodense mass approximately $2.7 \times 2.2 \mathrm{~cm}$ in size in the left lobe of the liver, the hepatic enhancement surrounding the abscesses having disappeared (short arrows).

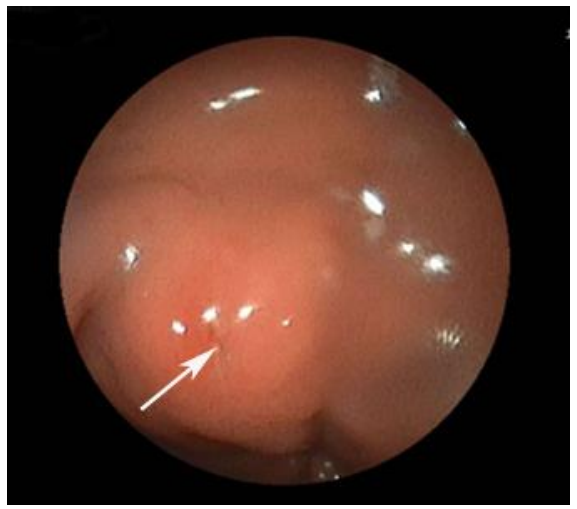

Fig. 2. Nasal endoscopy indicating an ulcer $0.9 \times 1.0 \mathrm{~cm}$ in size at the lesser curve of the gastric antrum (arrow). 


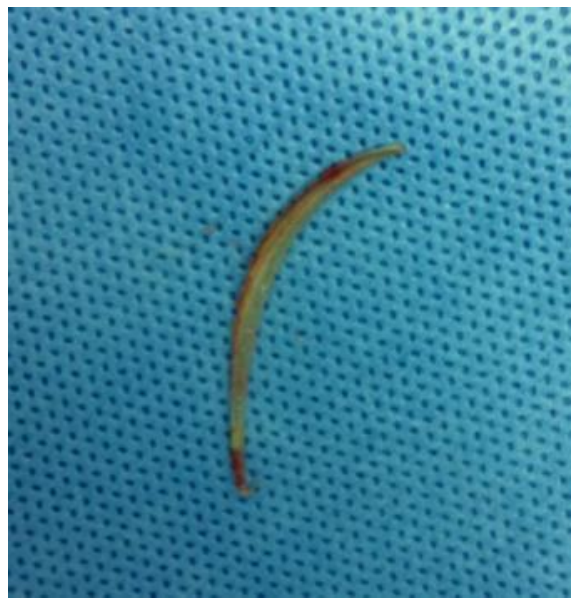

Fig. 3. The fish bone was removed surgically.

\section{References}

$>1$ Ng CT, Htoo A, Tan SY: Fish bone-induced hepatic abscess: medical treatment. Singapore Med J 2011;52:e56-e58.

-2 Santos SA, Alberto SC, Cruz E, et al: Hepatic abscess induced by foreign body: case report and literature review. World J Gastroenterol 2007;13:1466-1470.

-3 Chintamani, Singhal V, Lubhana P, et al: Liver abscess secondary to a broken needle migration - a case report. BMC Surg 2003;3:8.

$\checkmark 4$ Clarençon F, Scatton 0, Bruguière E, et al: Recurrent liver abscess secondary to ingested fish bone migration. Surg Today 2008;38:572-575.

5 Volk M, Geissler A, Herold T, et al: The computed tomographic demonstration of duodenal perforation caused by a fish bone. Rofo 1997;167:198-200.

6 Dugger K, Lebby T, Brus M, et al: Hepatic abscess resulting from gastric perforation of a foreign object. Am J Emerg Med 1990;8:323-325.

7 Hochman JS, Tamis JE, Thompson TD, et al: Sex, clinical presentation, and outcome in patients with acute coronary syndromes. N Engl J Med 1999;341:226-232. 